\title{
An efficient route for the synthesis of some monoazo disperse dyes derived from nicotinic acid derivatives
}

\author{
Abdulaziz Alnajjar a, Marzouq Alsaiedi a and Morsy Ahmed El-Apasery b,* \\ a Applied Science Department, College of Technological Studies, Public Authority for Applied Education and Training, Safat, 70654, Kuwait \\ b Dyeing, Printing and Textile Auxiliaries Department, Textile Research Division, National Research Centre, 12622 Dokki, Giza, Egypt \\ *Corresponding author at: Dyeing, Printing and Textile Auxiliaries Department, Textile Research Division, National Research Centre, 12622 Dokki, Giza, Egypt. \\ Tel.: +2.02.3371718; fax: +2.02.3370931. E-mail address: elapaserym@yahoo.com (M.A. El-Apasery).
}

\section{ARTICLE INFORMATION}

Received: 03 January 2013

Received in revised form: 04 February 2013

Accepted: 05 February 2013

Online: 31 March 2013

\section{KEYWORDS}

Enaminones

Nicotinic acid

Disperse dyes

Arylhydrazonals

Dye intermediates

Arylazonicotinates

\section{Introduction}

Every year, a number of dyes are introduced for coloring textile fibres by researchers and colorists. Only a few of those are commercialized, after passing necessary standards such as good build up, fastness properties and toxicity [1]. It is well known that disperse dyes are the most important group for dyeing of hydrophobic fibres, especially polyester.

The considerable biological and medicinal activities of nicotinic acid and its derivatives [2-5] have promoted recent interest in synthesis of new nicotinic acids of potential biological activities. Although condensation reactions of arylhydrazonals with active methylene nitriles were originally reported to afford pyridazin-6-imines [6], more recent studies have demonstrated that arylazonicotinates are also formed in some of these processes [7-9]. Because arylazonicotinates are a valuable class of arylazopyridine dyes whose chemistry has attracted some interest as new disperse dyes [10-12], it seemed of value to undertake an investigation aimed at exploring the potential utility of arylhydrazonals as precursors for the preparation of these targets.

In conjunction to our effort for synthesis poly-substituted nicotinates either as new antimicrobial agents or new disperse dye intermediates, we report herein, synthesis of new potential antimicrobial and disperse dyes with nicotinic acid skeleton via simple efficient routes. Also our investigation, described below, has led to the synthesis of different types of substances, including 2-aminoazonicotinate and 2-hydroxyazonicotate derivatives for utilizing as heterocyclic components for various disperse dyes.

\section{Experimental}

\subsection{Instrumentation}

Melting points are uncorrected. All melting points were recorded on a Griffin melting point apparatus and are reported uncorrected. IR spectra were recorded using $\mathrm{KBr}$ disks using a Perkin-Elmer System 2000 FT-IR spectrophotometer. ${ }^{1} \mathrm{H}$ NMR $(400 \mathrm{MHz})$ or $(600 \mathrm{MHz})$ and ${ }^{13} \mathrm{C}$ NMR $(100 \mathrm{MHz})$ or $(150 \mathrm{MHz})$ spectra were recorded at $25{ }^{\circ} \mathrm{C}$ in $\mathrm{CDCl}_{3}$ or DMSO- $d_{6}$ as solvent with TMS as internal standard on a Bruker DPX 400 or 600 super-conducting NMR spectrometer. Chemical shifts are reported in ppm. Mass spectra were measured using a high resolution GC-MS (DFS) Thermo spectrometer with EI (70 EV). Microanalyses were performed on a LECO CHNS-932 Elemental Analyzer. Compounds 10b, 10c, and $\mathbf{1 1 b}$ are prepared according to our previous work $[13,14]$. The crystal structure of compound 9 was determined by Bruker AXS X8 Prospector Single Crystal X-Ray Diffractometer at Kuwait University. The crystal was kept at 296(2) K during data collection. The structure was solved with the Program SHELXL-97 Software package.

\subsection{Ethyl-6-amino-5-cyano-4-phenyl-4H-pyran-3- carboxylate (7)}

A mixture of benzylidene malononitrile, 1a, $(1.54 \mathrm{~g}, 0.01$ mol), ethyl propiolate, 2, (1.0 g, $0.01 \mathrm{~mol}$ ) and L-proline, 3, $(10 \% \mathrm{~mol})$ was added together. The mixture was refluxed in absolute ethanol $(15 \mathrm{~mL})$ for $4 \mathrm{~h}$, followed by TLC. The crude compound formed was recrystallized from ethanol as white crystalline solid (Scheme 1). Yield: $65 \%$. M.p.: $227-230{ }^{\circ} \mathrm{C} .{ }^{1} \mathrm{H}$ NMR (400 MHz, DMSO- $\left.d_{6}, \delta, \mathrm{ppm}\right): 1.08\left(\mathrm{t}, 3 \mathrm{H}, J=7.2 \mathrm{~Hz}, \mathrm{CH}_{3}\right)$, $4.01\left(\mathrm{q}, 2 \mathrm{H}, J=7.2 \mathrm{~Hz}, \mathrm{CH}_{2}\right), 4.23(\mathrm{~s}, 1 \mathrm{H}$, pyran $\mathrm{H} 4), 7.03(\mathrm{~s}, 2 \mathrm{H}$, $\mathrm{NH}_{2}$ ) $, 7.17(\mathrm{~d}, 2 \mathrm{H}, J=8.0 \mathrm{~Hz}, \mathrm{Ar}-\mathrm{H}), 7.23(\mathrm{t}, 1 \mathrm{H}, J=8.0 \mathrm{~Hz}, \mathrm{Ar}-\mathrm{H})$, $7.32(\mathrm{t}, 2 \mathrm{H}, J=8.0 \mathrm{~Hz}, \mathrm{Ar}-\mathrm{H}), 7.71(\mathrm{~s}, 1 \mathrm{H}$, pyran $\mathrm{H} 6)$.

European Journal of Chemistry 

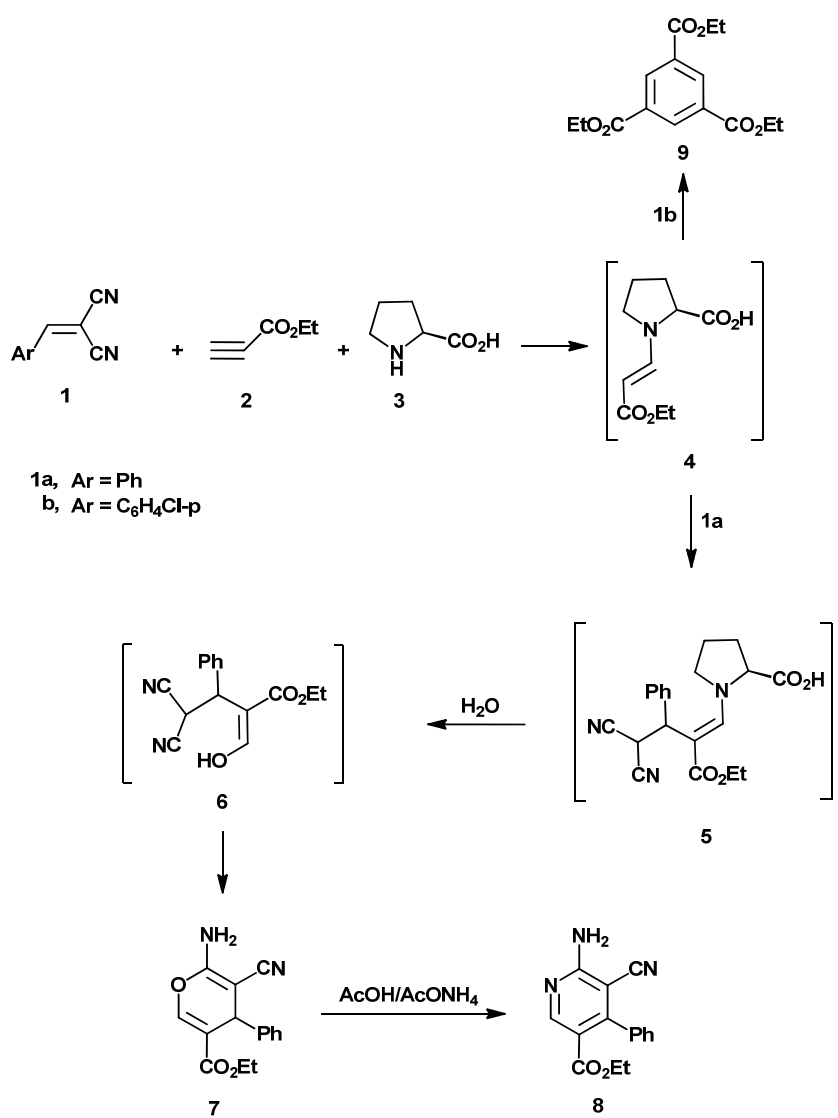

Scheme 1

${ }^{13} \mathrm{C}$ NMR (100 MHz, DMSO- $\left.d_{6}, \delta, \mathrm{ppm}\right): 14.33\left(\mathrm{CH}_{3}\right), 37.55$ (pyran C4), 57.79 (pyran C3), $60.70\left(\mathrm{CH}_{2}\right), 111.78$ (pyran C5), $120.16(\mathrm{CN}), 127.41,127.84,128.93,144.69,148.23,159.05$ (CO), 164.98 (pyran C6). MS (m/z, (\%)): $270\left(\mathrm{M}^{+}, 43\right), 271$ $\left(\mathrm{M}^{+}+1,8\right)$. Anal. calcd. for $\mathrm{C}_{15} \mathrm{H}_{14} \mathrm{~N}_{2} \mathrm{O}_{3}$ : C, 66.66; $\mathrm{H}, 5.22$; $\mathrm{N}$, 10.36. Found: C, 66.58; H, 5.29; N, 10.44\%.

\subsection{Ethyl-6-amino-5-cyano-4-phenylnicotinate (8)}

A solution of pyran, 7, $(0.70 \mathrm{~g}, 2.5 \mathrm{mmol})$ in acetic acid (10 $\mathrm{mL}$ ) containing ammonium acetate $(1 \mathrm{~g})$ was refluxed $5 \mathrm{~h}$, Then, the reaction mixture was cooled to room temperature. The solid which formed was collected by filtration, washed with water and recrystallized from ethanol as yellowish white crystalline solid (Scheme 1). Yield: 71\%. M.p.: $179-181^{\circ} \mathrm{C} .{ }^{1} \mathrm{H}$ NMR (400 MHz, DMSO- $d_{6}, \delta$, ppm): $0.87\left(\mathrm{t}, 3 \mathrm{H}, J=7.2 \mathrm{~Hz}, \mathrm{CH}_{3}\right)$, $3.92\left(\mathrm{q}, 2 \mathrm{H}, J=7.2 \mathrm{~Hz}, \mathrm{CH}_{2}\right), 7.28-7.30(\mathrm{~m}, 2 \mathrm{H}, \mathrm{Ar}-\mathrm{H}), 7.45-7.47$ (m, 3H, Ar-H), 7.7 (s, 2H, NH 2$), 8.67 \mathrm{ppm}(\mathrm{s}, 1 \mathrm{H}$, pyridyl $\mathrm{H} 6) .{ }^{13} \mathrm{C}$ NMR (100 MHz, DMSO-d $6, \delta, \mathrm{ppm}): 13.46\left(\mathrm{CH}_{3}\right), 60.17\left(\mathrm{CH}_{2}\right)$, 90.53 (pyridyl C3), 114.41 (pyridyl C5), 115.48 (CN), 127.72, 128.01, 128.61, 136.78, 155.02, 156.25, 161.66 (CO), 164.52 (pyridyl C6). MS (m/z, (\%)): $267\left(\mathrm{M}^{+}, 44\right), 268\left(\mathrm{M}^{+}+1,9\right)$. Anal. calcd. for $\mathrm{C}_{15} \mathrm{H}_{13} \mathrm{~N}_{3} \mathrm{O}_{2}$ : C, 67.41; $\mathrm{H}, 4.90 ; \mathrm{N}, 15.72$. Found: C, 67.54; H, 4.83; N, $15.81 \%$.

\subsection{Benzen -1,3,5-tricarboxylic acid triethyl ester (9)}

A mixture of $p$-chlorobenzylidene malononitrile, $\mathbf{1 b},(1.54$ g, $0.01 \mathrm{~mol})$, ethyl propiolate $(1.0 \mathrm{~g}, 0.01 \mathrm{~mol})$ and L-proline or DBU $(10 \% \mathrm{~mol})$ was added together. The mixture was refluxed in absolute ethanol $(15 \mathrm{~mL})$ for $8 \mathrm{~h}$, followed by TLC. The crude compound formed was recrystallized from ethanol as pale yellow crystalline solid (Scheme 1). Yield: 81\%. M.p.: 140-142 ${ }^{\circ} \mathrm{C} .{ }^{1} \mathrm{H}$ NMR (400 MHz, DMSO- $d_{6}, \delta$, ppm): $1.38(\mathrm{t}, 9 \mathrm{H}, J=7.2 \mathrm{~Hz}$, $\left.3 \mathrm{CH}_{3}\right), 4.41\left(\mathrm{q}, 6 \mathrm{H}, J=7.2 \mathrm{~Hz}, 3 \mathrm{CH}_{2}\right), 8.65(\mathrm{~s}, 3 \mathrm{H}, \mathrm{Ar}-\mathrm{H}) . \mathrm{MS}(\mathrm{m} / \mathrm{z}$ (\%)): $294\left(\mathrm{M}^{+}, 11\right), 295\left(\mathrm{M}^{+}+1,5\right.$.). Anal. calcd. for $\mathrm{C}_{15} \mathrm{H}_{18} \mathrm{O}_{6}: \mathrm{C}$ 61.22 ; H, 6.16. Found: C, 61.29; H, 6.19\%.

\subsection{3-Dimethylamino-1-naphthalen-2-yl-propenone (10a)}

A mixtures of 2-acetylnaphthalen $(10 \mathrm{mmol})$ and $\mathrm{N}, \mathrm{N}$ dimethylformamide-dimethylacetal (DMF-DMA) $(15 \mathrm{mmol})$ were stirred at reflux for $24 \mathrm{~h}$. The separated solid product obtained on standing at room temperature was collected by filtration, washed by EtOH and recrystallized from $\mathrm{EtOH}$ to afford the corresponding enaminone, 10a, as yellow crystals (Scheme 2). Yield: 85\%. M.p.: 92-94 ${ }^{\circ} \mathrm{C}$. FT-IR $\left(\mathrm{KBr} \mathrm{cm}^{-1}\right): 1639$ (CO). ${ }^{1} \mathrm{H}$ NMR (400 MHz, DMSO- $\left.d_{6}, \delta, \mathrm{ppm}\right): 2.88\left(\mathrm{~s}, 3 \mathrm{H}, \mathrm{CH}_{3}\right)$, $3.11\left(\mathrm{~s}, 3 \mathrm{H}, \mathrm{CH}_{3}\right), 5.84(\mathrm{~d}, 1 \mathrm{H}, J=12 \mathrm{~Hz}$, olefinic $\mathrm{CH}), 7.41-7.50$ $(\mathrm{m}, 3 \mathrm{H}, \mathrm{Ar}-\mathrm{H}), 7.76(\mathrm{~d}, 1 \mathrm{H}, J=12 \mathrm{~Hz}$, olefinic $\mathrm{CH}), 7.91-7.93(\mathrm{~m}$ 4H, Ar-H). MS (m/z, (\%)): $225\left(\mathrm{M}^{+}, 66\right), 226\left(\mathrm{M}^{+}+1,8\right)$. Anal. calcd. for $\mathrm{C}_{15} \mathrm{H}_{15} \mathrm{NO}$ : $\mathrm{C}, 79.97 ; \mathrm{H}, 6.71 ; \mathrm{N}, 6.22$. Found: $\mathrm{C}, 80.05$; $\mathrm{H}, 6.77$; N, 6.18\%.

\subsection{General procedure for the preparation of compounds (11a,c)}

Cold solution of benzenediazonium chloride $(10 \mathrm{mmol})$ was prepared by adding a solution of sodium nitrite (1.4 $\mathrm{g}$ dissolved in $10 \mathrm{~mL}$ water) to cold solution of primary aromatic amines hydrochloride ( $10 \mathrm{mmol}$ in $10 \mathrm{~mL}, 6 \mathrm{M} \mathrm{HCl})$ with stirring. The resulting solution of benzenediazonium chloride was then added to a cold solution of the enaminones, $10 a$ or $\mathbf{1 0 c},(10$ $\mathrm{mmol})$ in ethanol $(50 \mathrm{~mL})$ in the presence of sodium acetate $(4.2 \mathrm{~g}, 30 \mathrm{mmol})$. 


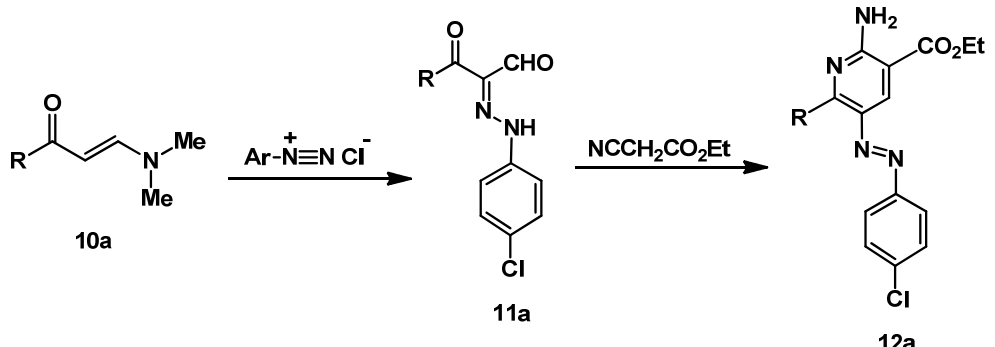

10-12a, R = naphthalene-2-yl, Ar = p-chlorophenyl

Scheme 2

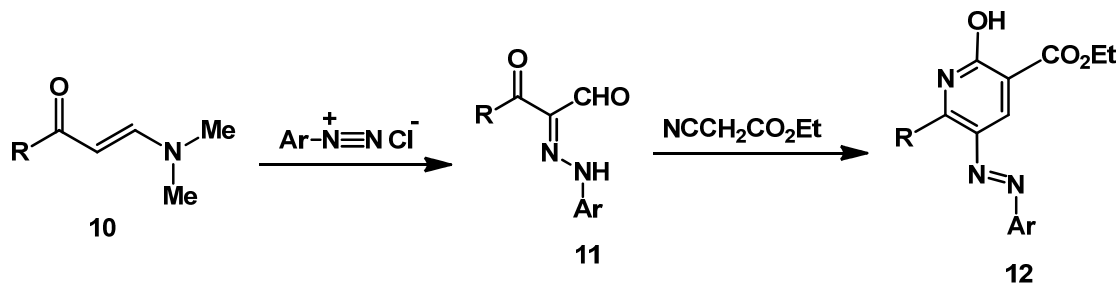

10-12b, R = 1H-pyrrol-2-yl, $\mathrm{Ar}=\mathrm{Ph}$

c, $R=$ pyrazin-2-yl, Ar $=p$-tolyl

Scheme 3

The reaction mixture was stirred at room temperature for 1 h. The formed solid product was collected by filtration and washed with water then recrystallized from EtOH to afford compound 11a or 11c, respectively.

2-[(4-Chlorophenyl)hydrazono]-3-naphthalen-2-yl-3oxopropionaldehyde (11a): Yellow crystals (Scheme 2). Yield: 74\%. M.p.: 128-130 ${ }^{\circ}$ C. FT-IR (KBr cm$\left.{ }^{-1}\right): 3431(\mathrm{NH}), 1637$ (CO). ${ }_{1}^{1} \mathrm{H}$ NMR (400 MHz, DMSO- $\left.d_{6}, \delta, \mathrm{ppm}\right): 7.41-7.52(\mathrm{~m}, 4 \mathrm{H}, \mathrm{Ar}-\mathrm{H})$, 7.62-7.73 (m, 2H, Ar-H), 7.92-8.54 (m, 5H, Ar-H), 10.06 (s, 1H, $\mathrm{NH}), 14.16(\mathrm{~s}, 1 \mathrm{H}, \mathrm{CHO})$. MS (m/z, (\%)): $336\left(\mathrm{M}^{+}, 38\right), 337$ $\left(\mathrm{M}^{+}+1\right.$, 7.75). Anal. calcd. for $\mathrm{C}_{19} \mathrm{H}_{13} \mathrm{ClN}_{2} \mathrm{O}_{2}$ : C, 67.76; $\mathrm{H}, 3.89$; $\mathrm{N}$, 8.32.Found: C, 67.71; H, 3.95; N, 8.44\%

3-Oxo-3-(pyrazin-2-yl)-2-(2-p-tolylhydrazono)propanal (11c): Wine red crystals (Scheme 3). Yield: 78\%. M.p.: 140-141 ${ }^{\circ}$ C. FT-IR (KBr cm${ }^{-1}$ ): 3119 (NH), 1657, 1642 (CO). ${ }^{1} \mathrm{H}$ NMR (400 MHz, DMSO- $\left.d_{6}, \delta, \mathrm{ppm}\right): 2.28\left(\mathrm{~s}, 3 \mathrm{H}, \mathrm{CH}_{3}\right), 7.20(\mathrm{~d}, 2 \mathrm{H}, J=6.8 \mathrm{~Hz}$, Ar-H), 7.29 (d, 2H, $J=5.6 \mathrm{~Hz}, \mathrm{Ar}-\mathrm{H}), 8.81-8.85(\mathrm{~m}, 2 \mathrm{H}, \mathrm{Ar}-\mathrm{H})$, 9.04 (s, 1H, Ar-H); 10.02 (s, 1H, NH), 14.36 (s, 1H, CHO). ${ }^{13} \mathrm{C}$ NMR (100 MHz, DMSO- $\left.d_{6}, \delta, \mathrm{ppm}\right): 20.8\left(\mathrm{CH}_{3}\right), 117.0,130.0$, $131.7,136.3,138.9,143.7,144.7,145.0,150.8,187.0,188.7$. MS (m/z, (\%)): 268 ([M]+, 20). Anal. calcd. for $\mathrm{C}_{14} \mathrm{H}_{12} \mathrm{~N}_{4} \mathrm{O}_{2}: \mathrm{C}, 62.68$; H, 4.51; N, 20.88;. Found: C, 62.88; H, 4.82; N, 20.57\%.

\subsection{Ethyl 2-amino-5-((4-chlorophenyl)diazenyl)-6- (naphthalen-2-yl)nicotinate (12a)}

A mixture of the arylhydrazonalas, 11a, $(10 \mathrm{mmol})$, ethyl cyanoacetate $(1.2 \mathrm{~g}, 10 \mathrm{mmol})$ and ammonium acetate $(2 \mathrm{~g})$ in acetic acid $(30 \mathrm{~mL})$ was refluxed for $2 \mathrm{~h}$. then allowed to cool down to room temperature and poured onto ice cold water. The formed precipitate was collected by filtration washed with water and recrystallized from ethanol as orange crystals (Scheme 2). Yield: $77 \%$. M.p.: $89-90^{\circ} \mathrm{C}$. FT-IR $\left(\mathrm{KBr} \mathrm{cm}^{-1}\right): 3444$, $4350\left(\mathrm{NH}_{2}\right), 1743(\mathrm{CO}) .{ }^{1} \mathrm{H}$ NMR (400 MHz, DMSO- $\left.d_{6}, \delta, \mathrm{ppm}\right):$ 1.37 (t, 3H, $\left.J=7.4 \mathrm{~Hz}, \mathrm{CH}_{3}\right), 4.39$ (q, $\left.2 \mathrm{H}, J=7.4 \mathrm{~Hz}, \mathrm{CH}_{2}\right)$, 7.56$7.62(\mathrm{~m}, 4 \mathrm{H}, \mathrm{Ar}-\mathrm{H}), 7.72(\mathrm{~d}, 2 \mathrm{H}, J=8.0 \mathrm{~Hz}, \mathrm{Ar}-\mathrm{H}), 7.93-8.02(\mathrm{~m}$, $4 \mathrm{H}, \mathrm{Ar}-\mathrm{H}), 8.12\left(\mathrm{br}, 2 \mathrm{H}, \mathrm{NH}_{2}\right), 8.35(\mathrm{~s}, 1 \mathrm{H}, \mathrm{Ar}-\mathrm{H}), 8.62(\mathrm{~s}, 1 \mathrm{H}$, pyridine $\mathrm{H}) .{ }^{13} \mathrm{C}$ NMR $\left(100 \mathrm{MHz}, \mathrm{DMSO}-d_{6}, \delta, \mathrm{ppm}\right): 14.2\left(\mathrm{CH}_{3}\right)$, $61.2\left(\mathrm{CH}_{2}\right), 105.1,124.0,126.5,126.6,127.2,127.4,127.5$,
$128.2,128.6,129.4,129.5,131.0,132.2,133.1,134.6,134.9$ 136.6, 150.9, 159.8, 166.2 (CO). MS ( $\mathrm{m} / \mathrm{z},(\%)): 430\left(\mathrm{M}^{+}, 100\right)$, $431\left(\mathrm{M}^{+}+1,55\right)$. Anal. calcd. for $\mathrm{C}_{24} \mathrm{H}_{19} \mathrm{ClN}_{4} \mathrm{O}_{2}$ : C, 66.90; $\mathrm{H}, 4.44$; N, 13.00. Found: C, 66.88; H, 4.38; N, 13.11\%.

\subsection{General procedure for the synthesis of compounds (12b,c and 14)}

Independent mixtures of compound $\mathbf{1 1 b}$ or $\mathbf{1 1 c}(0.01 \mathrm{~mol})$, ethyl cyanoacetate or cyanoacetamide $(0.01 \mathrm{~mol})$, and ammonium acetate $(0.5 \mathrm{~g})$ in acetic acid $(10 \mathrm{~mL})$ was stirred at reflux for $30 \mathrm{~min}$ (progress of the reactions was monitored by using TLC using ethyl acetate: petroleum ether (1:1). The mixtures were cooled and then poured into ice-water. The solids that formed were collected by using filtration and crystallized from ethanol to give compound $\mathbf{1 2 b}, \mathbf{c}$ and 14

Ethyl 2-hydroxy-5-(phenyldiazenyl)-6-(1H-pyrrol-2-yl)nicotinate (12b): Dark brown powder (Scheme 3). Yield: 60\%. M.p.: 202-204 ${ }^{\circ} \mathrm{C}$. FT-IR (KBr cm$\left.{ }^{-1}\right): 3300(\mathrm{OH}), 3064(\mathrm{NH})$, 1598 (CO). ${ }^{1} \mathrm{H}$ NMR (400 MHz, DMSO- $\left.d_{6}, \delta, \mathrm{ppm}\right): 1.30(\mathrm{t}, 3 \mathrm{H}, J=$ $\left.7.2 \mathrm{~Hz}, \mathrm{CH}_{3}\right), 4.31\left(\mathrm{q}, 2 \mathrm{H}, J=7.2 \mathrm{~Hz}, \mathrm{CH}_{2}\right), 7.10-7.77(\mathrm{~m}, 8 \mathrm{H}, \mathrm{Ar}-$ $\mathrm{H}), \quad 8.31\left(\mathrm{~s}, 1 \mathrm{H}\right.$, pyridyl-H); $11.89\left(\mathrm{~s}, 1 \mathrm{H}, \quad \mathrm{NH}, \quad \mathrm{D}_{2} \mathrm{O}\right.$ exchangeable)., 12.18 (s, $1 \mathrm{H}, \mathrm{OH}, \mathrm{D}_{2} \mathrm{O}$ exchangeable). ${ }^{13} \mathrm{C}$ NMR (100 MHz, DMSO- $\left.d_{6}, \delta, \mathrm{ppm}\right): 13.9\left(\mathrm{CH}_{3}\right), 61.7\left(\mathrm{CH}_{2}\right), 110.9$, 117.2, 121.2, 123.0, 126.0, 127.5, 128.8, 129.0, 130.9, 131.4, 141.3, 141.8, 175.0, 176.9 (CO). MS ( $\mathrm{m} / \mathrm{z},(\%)): 337\left([\mathrm{M}+1]^{+}\right.$, 95). Anal. calcd. for $\mathrm{C}_{18} \mathrm{H}_{16} \mathrm{~N}_{4} \mathrm{O}_{3}$ : C, 64.28; $\mathrm{H}, 4.79 ; \mathrm{N}, 16.66$ Found: C, 63.97; $\mathrm{H}, 4.63$; $\mathrm{N}, 16.44 \%$. HRMS: $\mathrm{m} / \mathrm{z}$ (EI) for $\mathrm{C}_{18} \mathrm{H}_{16} \mathrm{~N}_{4} \mathrm{O}_{3}$; calcd. 336.1216; found: 336.1216.

Ethyl 2-hydroxy-6-(pyrazin-2-yl)-5-(p-tolyldiazenyl)nicotinate (12c): Dark red powder (Scheme 3). Yield: 68\%. M.p.: $>300{ }^{\circ} \mathrm{C}$. FT-IR $\left(\mathrm{KBr} \mathrm{cm}^{-1}\right): 3312(\mathrm{OH}), 1610(\mathrm{CO}) .{ }^{1} \mathrm{H}$ NMR (400 MHz, DMSO- $d_{6}, \delta, \mathrm{ppm}$ ): $1.30\left(\mathrm{t}, 3 \mathrm{H}, J=7.2 \mathrm{~Hz}, \mathrm{CH}_{3}\right.$ ), 2.33 (s, $\left.3 \mathrm{H}, \mathrm{CH}_{3}\right), 4.23\left(\mathrm{q}, 2 \mathrm{H}, J=7.2 \mathrm{~Hz}, \mathrm{CH}_{2}\right), 7.06-7.77(\mathrm{~m}, 6 \mathrm{H}, \mathrm{Ar}-$ $\mathrm{H}), 8.16$ (s, 1H, Ar-H), 9.04 (s, 1H, arom-H), 12.00 (s, 1H, OH, $\mathrm{D}_{2} \mathrm{O}$ exchangeable). ${ }^{13} \mathrm{C}$ NMR (100 MHz, DMSO- $\left.d_{6}, \delta, \mathrm{ppm}\right): 13.9$ $\left(\mathrm{CH}_{3}\right), 20.7\left(\mathrm{CH}_{3}\right), 55.8\left(\mathrm{CH}_{2}\right), 112.6,117.1,121.3,123.2,125.6$ 127.5, 128.8, 129.2, 132.4, 135.0, 139.9, 157.5, 161.9, 165.7 (CO). 


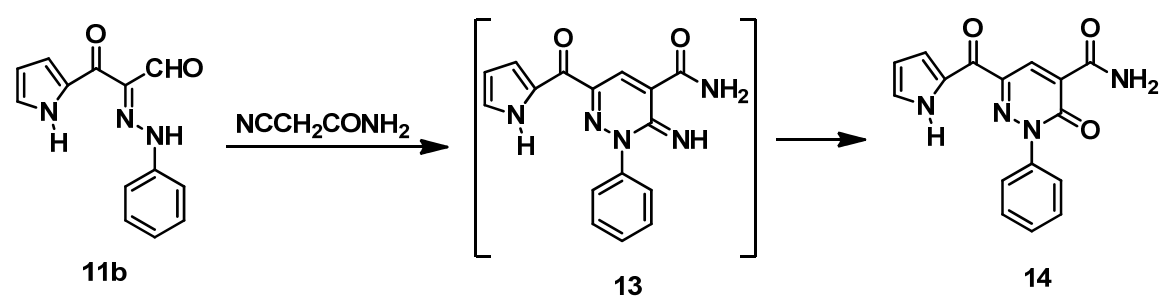

Scheme 4

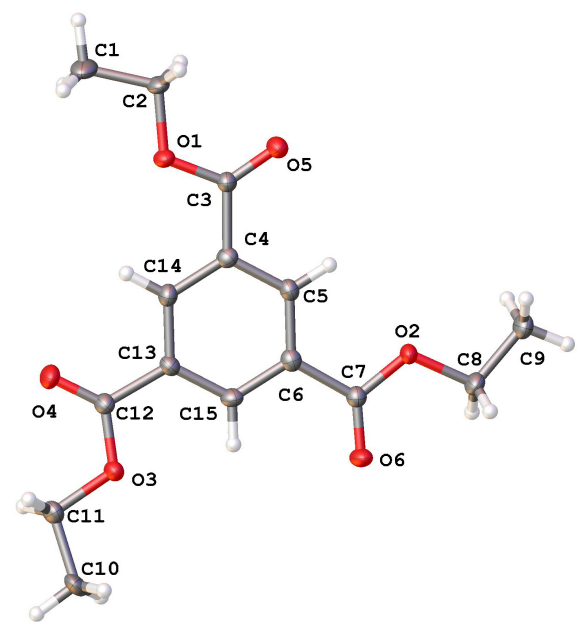

Figure 1. X-ray crystal structure of compound 9.

MS (m/z, (\%)):363 (M+). Anal. calcd. for $\mathrm{C}_{19} \mathrm{H}_{17} \mathrm{~N}_{5} \mathrm{O}_{3}$ : C, 62.80; $\mathrm{H}$, 4.72; N, 19.27. Found: C, 62.55; H, 4.65; N, $19.16 \%$.

3-0xo-2-phenyl-6-(1H-pyrrole-5-carbonyl)-2,3-dihydro pyridazine-4-carboxamide (14): Brown powder (Scheme 4). Yield: $88 \%$. M.p.: $250-252^{\circ} \mathrm{C}$. FT-IR $\left(\mathrm{KBr} \mathrm{cm}^{-1}\right): 3372,3301$ $\left(\mathrm{NH}_{2}\right), 3063(\mathrm{NH}), 1697$ (CO), 1595 (CO). ${ }^{1} \mathrm{H}$ NMR (400 MHz, DMSO- $\left.d_{6}, \delta, \mathrm{ppm}\right): 6.25(\mathrm{~s}, 1 \mathrm{H}, \mathrm{Ar}-\mathrm{H}), 7.09-7.66(\mathrm{~m}, 4 \mathrm{H}, \mathrm{Ar}-\mathrm{H})$, $7.68(\mathrm{~d}, 2 \mathrm{H}, J=8.0 \mathrm{~Hz} \mathrm{Ar}-\mathrm{H}), 7.76(\mathrm{~d}, 1 \mathrm{H}, J=8.4 \mathrm{~Hz} \mathrm{Ar}-\mathrm{H}), 8.59$ (s, 1H, pyridazinyl-H), 11.90 (s, $1 \mathrm{H}, \mathrm{NH}), 12.22$ (s, 2H, $\mathrm{NH}_{2}, \mathrm{D}_{2} \mathrm{O}$ exchangeable). ${ }^{13} \mathrm{C}$ NMR $\left(100 \mathrm{MHz}, \mathrm{DMSO}-d_{6}, \delta, \mathrm{ppm}\right): 110.66$, 117.2, 118.8, 121.3, 126.1, 127.6, 129.0, 131.8, 141.3, 149.5, 159.5, 162.2, 175.1, 176.9. MS ( $\mathrm{m} / \mathrm{z},(\%)): 308\left([\mathrm{M}]^{+}, 100\right)$. Anal. calcd. for $\mathrm{C}_{16} \mathrm{H}_{12} \mathrm{~N}_{4} \mathrm{O}_{3}: 62.33 ; \mathrm{H}, 3.92 ; \mathrm{N}, 18.17$. Found: 62.41; H, 4.19; $\mathrm{N}, 18.32 \%$. HRMS: $m / z$ (EI) for $\mathrm{C}_{16} \mathrm{H}_{12} \mathrm{~N}_{4} \mathrm{O}_{3}$; calcd. 308.0904; found: 308.0904 .

\section{Results and discussion}

Similar to recent report [15], ethyl propiolate (2), and benzylidenemalononitrile (1), reacted in ethanolic solution in the presence of catalytic amount of L-proline, as a catalyst, yielded the 2-aminopyran, 7 , in $65 \%$ yield. It is believed that compound $\mathbf{2}$ initially add L-proline to yield compound $\mathbf{4}$ that then reacted with benzylidenemalononitrile yielding the acyclic intermediate, 5, that then afford compound $\mathbf{6}$ that cyclized into the pyran, 7 . The 2 -aminopyran, 7 , readily rearranged into the 2-aminonicotinate, 8 , when refluxed in acetic acid in the presence of ammonium acetate (Scheme 1).

Thus trials to extend this approach for synthesis of other substituted nicotinates, $\mathbf{8}$, via reacting compound $\mathbf{1 b}$ with compound 2 utilizing the same procedure failed. As instead of formation of compound $\mathbf{7 b}$ the 1,3,5-trisubstituted benzoate, $\mathbf{9}$, was formed and whose structure could be confirmed by the Xray crystal structure determination (Figure 1). Crystal data and structure refinement for compound $\mathbf{9}$ are listed in Table 1 . Bond distances and angles calculated from the final atomic coordinates are given in Table 2 and 3, respectively.

Table 1. Crystal data and structure refinement for compound $\mathbf{9}$.

\begin{tabular}{|c|c|}
\hline Empirical formula & $\mathrm{C}_{15} \mathrm{H}_{18} \mathrm{O}_{6}$ \\
\hline Formula weight & 294.29 \\
\hline Temperature/K & $296(2)$ \\
\hline Crystal system & Hexagonal \\
\hline Space group & $\mathrm{P} 66_{5}$ \\
\hline $\mathrm{a} / \AA ̊$ & $11.3311(5)$ \\
\hline $\mathrm{b} / \AA ̊$ & $11.3311(5)$ \\
\hline$c / \AA$ & $19.5375(10)$ \\
\hline$\alpha /^{\circ}$ & 90 \\
\hline$\beta /^{\circ}$ & 90 \\
\hline$\gamma /{ }^{\circ}$ & 120 \\
\hline Volume $/ \AA^{3}$ & $2172.42(18)$ \\
\hline Z & 6 \\
\hline$\rho_{\text {calc }} \mathrm{mg} / \mathrm{mm}^{3}$ & 1.350 \\
\hline $\mathrm{m} / \mathrm{mm}^{-1}$ & 0.879 \\
\hline $\mathrm{F}(000)$ & 936.0 \\
\hline Crystal size $/ \mathrm{mm}^{3}$ & $0.34 \times 0.07 \times 0.04$ \\
\hline $2 \Theta$ range for data collection & 9.02 to $132.96^{\circ}$ \\
\hline Index ranges & $-12 \leq \mathrm{h} \leq 10,-10 \leq \mathrm{k} \leq 12,-23 \leq \mathrm{l} \leq 21$ \\
\hline Reflections collected & 4546 \\
\hline Independent reflections & $2271[\mathrm{R}$ (int) $=0.0921]$ \\
\hline Data/restraints/parameters & $2271 / 1 / 193$ \\
\hline Goodness-of-fit on $\mathrm{F}^{2}$ & 1.064 \\
\hline Final $R$ indexes $[I>=2 \sigma(I)]$ & $\mathrm{R}_{1}=0.0686, \mathrm{wR}_{2}=0.1650$ \\
\hline Final $\mathrm{R}$ indexes [all data] & $\mathrm{R}_{1}=0.0779, \mathrm{w} \mathrm{R}_{2}=0.1691$ \\
\hline Largest diff. peak/hole / e $\AA^{-3}$ & $0.41 /-0.38$ \\
\hline Flack parameter & $-0.1(3)$ \\
\hline
\end{tabular}

In an alternative synthetic methodology ethyl 2-amino- or ethyl 2-hydroxyazonicotinate disperse dyes, 12a-c, could be synthesized. Thus compounds 10a-c coupled with aryldiene diazonium chloride to yield compounds 11a-c. Compound 11a condensed with ethyl cyanoacetate to yield ethyl 2aminoazonicotinate disperse dye, 12a (Scheme 2). 
Table 2. Bond lengths for compound 9.

\begin{tabular}{llllll}
\hline Atom & Atom & Length, $\AA$ & Atom & Atom & Length, $\AA$ \\
\hline 01 & C2 & $1.456(3)$ & C4 & C5 & $1.394(4)$ \\
01 & C3 & $1.336(3)$ & C4 & C14 & $1.385(4)$ \\
O2 & C7 & $1.338(3)$ & C5 & C6 & $1.392(4)$ \\
O2 & C8 & $1.457(3)$ & C6 & C7 & $1.499(4)$ \\
O3 & C11 & $1.458(3)$ & C6 & C15 & $1.383(4)$ \\
O3 & C12 & $1.339(3)$ & C8 & C9 & $1.495(4)$ \\
04 & C12 & $1.206(4)$ & C10 & C11 & $1.499(4)$ \\
05 & C3 & $1.205(3)$ & C12 & C13 & $1.495(4)$ \\
O6 & C7 & $1.208(3)$ & C13 & C14 & $1.395(4)$ \\
C1 & C2 & $1.488(4)$ & C13 & C15 & $1.390(4)$ \\
C3 & C4 & $1.497(4)$ & & & \\
\hline
\end{tabular}

Table 3. Bond angles for compound 9.

\begin{tabular}{llllllll}
\multicolumn{1}{l}{ Atom } & Atom & Atom & Angle, $^{\circ}$ & Atom & Atom & Atom & Angle, $^{\circ}$ \\
\hline C3 & O1 & C2 & $116.1(2)$ & O2 & C7 & C6 & $111.9(2)$ \\
C7 & O2 & C8 & $116.8(2)$ & O6 & C7 & O2 & $124.0(3)$ \\
C12 & O3 & C11 & $115.9(2)$ & O6 & C7 & C6 & $124.1(3)$ \\
01 & C2 & C1 & $107.3(2)$ & O2 & C8 & C9 & $107.2(2)$ \\
01 & C3 & C4 & $111.6(2)$ & O3 & C11 & C10 & $107.3(3)$ \\
05 & C3 & O1 & $124.5(3)$ & O3 & C12 & C13 & $111.9(2)$ \\
05 & C3 & C4 & $123.9(3)$ & O4 & C12 & O3 & $124.3(2)$ \\
C5 & C4 & C3 & $118.3(2)$ & O4 & C12 & C13 & $123.8(3)$ \\
C14 & C4 & C3 & $121.7(2)$ & C14 & C13 & C12 & $117.9(2)$ \\
C14 & C4 & C5 & $120.0(2)$ & C15 & C13 & C12 & $122.8(3)$ \\
C6 & C5 & C4 & $119.7(3)$ & C15 & C13 & C14 & $119.4(3)$ \\
C5 & C6 & C7 & $121.4(3)$ & C4 & C14 & C13 & $120.4(2)$ \\
C15 & C6 & C5 & $120.2(3)$ & C6 & C15 & C13 & $120.4(2)$ \\
C15 & C6 & C7 & $118.4(2)$ & & & & \\
\hline
\end{tabular}

In contrast, when the condensation reaction of compound 11b or 11c with ethyl cyanoacetate are conducted in the presence of a catalytic amount of ammonium acetate, ethyl 2hydroxyazonicotinate, $\mathbf{1 2 b}$ or $\mathbf{1 2 c}$, disperse dyes are produced (Scheme 3).

In the final phase of the current effort, we observed that reactions of compound $\mathbf{1 1 b}$ with cyanoacetamide in the presence of ammonium acetate in acetic acid for one hour lead to the respective pyridazinone, 14, which is likely formed via the intermediacy of the readily hydrolyzed imine analogs, 13 (Scheme 4).

Currently, we are utilizing the 2-amino- and 2- hydroxylazonicotinates disperse dyes for dyeing polyester fabrics by using high temperature dyeing method. We are also inspecting the biological activity of these disperse dyes against Grampositive bacteria, Gram-negative bacteria and yeast

\section{Conclusion}

In conclusion, in the investigation described above, a series of arylazonicotinates disperse dyes were synthesized in a good yields via condensation of arylhydrazonals with active methylenes.

\section{Acknowledgements}

This research was done by the financial support of the Public Authority for Applied Education and Training (Transform grant TS-07-11) of Kuwait.

\section{Supplementary material}

CCDC-800579 of compound 9 contains the supplementary crystallographic data for this paper. These data can be obtained free of charge via www.ccdc.cam.ac.uk/data_request/cif, or by e-mailing data_request@ccdc.cam.ac.uk, or by contacting The Cambridge Crystallographic Data Centre, 12 Union Road, Cambridge CB2 1EZ, UK; fax: +44(0)1223-336033.

\section{References}

[1]. Sayed, A. Z.; Aboul-Fetouh, M. S.; Nassar, H. S. J. Mol. Struct. 2012, 1010, 146-151.
[2]. Gharanjig, K.; Sadeghi-Kiakhani, M.; Arami, M.; Mahmoodi, N. M.; Khosravi, A. Color. Technol. 2010, 126(1), 37-41.

[3]. Suesat, J.; Mungmeechai, T.; Suwanruji, P.; Parasuk, W.; Taylor, J. A Phillips, D. A. S. Color. Technol. 2011, 127(4), 217-222.

[4]. El-Apasery, M. A.; Al-Mousawi, S. M.; Mahmoud, H.; Elnagdi, M. H. Eur J. Chem. 2011, 2(2), 168-172.

[5]. Khalil, K.; Al-Matar, H.; Elnagdi, M. H. Eur. J. Chem. 2010, 1(4), 252258.

[6]. Behbehani, H.; Abdel-Khalik, M. M.; Elnagdi, M. H. Org. Prep. Proced. Int. 1999, 31(5), 551-557.

[7]. Al-Mousawi, S. M.; El-Apasery, M. A.; Mahmoud, H. M. Molecules 2012, 17, 11495-11506.

[8]. Al-Mousawi, S. M.; El-Apasery, M. A. Molecules 2012, 17, 6547-6556.

[9]. Al-Mousawi, S. M.; El-Apasery, M. A.; Mahmoud, H.; Elnagdi, M. H. Int Res. J. Pure Appl. Chem. 2012, 2, 77-90.

[10]. Kim, K. R.; Rhee, S. D.; Kim, H. Y.; Jung, W. H.; Yang, S. D.; Kim, S. S Ahn, J. H.; Cheon, H. Eur. J. Pharm. 2005, 518(1), 63-70.

[11]. Zaky, O. S.; Moustafa, M. S.; Selim, M. A.; El-Maghraby, A. M.; Elnagdi, M. H. Molecules 2012, 17(5), 5924-34.

[12]. Mohanta, G. P.; Manna, P. K.; Manavalan, R. Ind. J. Pharm. Sci. 2002, 64(5), 489-491.

[13]. El-Apasery, M. A.; Al-Mousawi, S. M.; Mahmoud, H.; Elnagdi, M. H. Int. Res. J. Pure Appl. Chem. 2011, 1, 69-93.

[14]. Bazhin, D. N.; Kudyakova, Y. S.; Burgart, Y. V.; Saloutin, V. I. Tetrahedron Lett. 2012, 53(15), 1961-1963.

[15]. Elnagdi, N. M. H.; Al-Hokbany, N. S. Molecules 2012, 17, 4300-4312. 